Dossier: Le Sfide Teologiche del Pluralismo Religioso - Articolo originale (c) (i)

DOI - 10.5752/P.2175-5841.2015v13n40p1790

\title{
Le interpellanze del pluralismo religioso nel contesto occidentale
}

\author{
Interpellations of religious pluralism in the Western context \\ Interpelações do pluralismo religioso no contexto ocidental
}

Marco Dal Corso*

\section{Sommario}

La realtà del pluralismo religioso interroga i diversi contesti culturali non potendo misconoscerne la storia, le dinamiche, la complessità specifica. Questo vale, evidentemente, anche per il contesto occidentale e in particolare, in questo nostro articolo, per quello italiano che, come vedremo, "vanta" peculiarità e dinamiche storiche specifiche rispetto ad altri parti del mondo occidentale. Si tratta di capire le interpellanze del pluralismo religioso per il futuro delle culture religiose, e non, che appartengono all'universo occidentale. L'articolo intende intrepretare la realtà del pluralismo religioso nel contesto occidentale e in quello specificatamente italiano, volendo leggere le note di tipo socio-antropologico come interpellanze per la teologia del dialogo interreligioso. Gli appelli del pluralismo, infatti, diventano argomento teologico solo dentro una teologia post-moderna del dialogo, di cui l'autore prova a dare una breve descrizione come grammatica religiosa della diversità, evidenziando come categorie principali quelle della gratuità e dell'ospitalità.

Parole chiave: Pluralismo religioso. Contesti culturali. Diversità. Culture religiose. Teologia postmoderna

\begin{abstract}
The reality of religious pluralism questions the different cultural contexts. In that regard we can not underestimate its history, dynamics and specific complexity. This applies of course also to the Western context. In this article, it also applies to the Italian context, which, as we shall see, has specific historical peculiarities and dynamics in relation to other parts of the Western world. This article aims to interpret the situation of religious pluralism in the Western context, specifically in the Italian context, seeking to understand the social anthropological observations as interpellations for the theology of inter-religious dialogue. The appeals of pluralism become, in fact, theological arguments only in a postmodern theology of dialogue, which the author tries to give a brief description as religious grammar of diversity, highlighting main categories of generosity and hospitality.
\end{abstract}

Keywords: Religious pluralism. Cultural contexts. Diversity. Religious culture. Postmodern theology

\section{Resumo}

A realidade do pluralismo religioso problematiza os diferentes contextos culturais, não podendo subestimar sua história, dinâmica e complexidade específica. Isto aplica-se, evidentemente, também para o contexto ocidental e em particular, em nosso artigo, para o contexto italiano que, como veremos, possui peculiaridades e dinâmica históricas específicas em relação às outras partes do mundo ocidental. 0 artigo se propõe a interpretar a realidade do pluralismo religioso no contexto ocidental, mais especificamente no contexto italiano, buscando entender as observações sócio antropológicas como interpelações para a teologia do diálogo inter-religioso. Os apelos do pluralismo tornam-se, de fato, argumentos teológicos apenas em uma teologia pós-moderna do diálogo, da qual o autor tenta dar uma breve descrição como gramática religiosa da diversidade, destacando-se como principais as categorias da generosidade e da hospitalidade.

Palavras-chave: pluralismo religioso. Contextos culturais. Diversidade. Culturas religiosas. Teologia pósmoderna

Articolo ricevuto in 18 set. 2015 e ha approvato il 03 dic.2015.

"Ph.d in teologia morale presso l'Università Misericorde, Fribourg (Svizzera) e quello in teologia sistematica a indirizzo ecumenico presso la Facoltà Valdese di teologia in Roma. Paese di origine: Itália. E mail: dalcorsomarco@tiscali.it 


\section{Introduzione}

La realtà del pluralismo religioso interroga i diversi contesti culturali non potendo misconoscerne la storia, le dinamiche, la complessità specifica. Questo vale, evidentemente, anche per il contesto occidentale e in particolare, in questo nostro articulo, per quello italiano che, come vedremo, "vanta" peculiarità e dinamiche storiche specifiche rispetto ad altri parti del mondo occidentale. Si tratta di capire le interpellanze del pluralismo religioso per il futuro delle culture religiose, e non, che appartengono all'universo occidentale. Alla scuola di Panikkar, infatti, abbiamo imparato che occorre riconoscere non tanto le sfide, quanto le interpellanze poste dal fenomeno multi-religioso odierno: abbiamo, cioè, lasciato l'arena del conflitto tra modernità e religione, dove valgono le sfide tra i contendenti, e abbiamo deciso di abitare l'agorà di tutti, dove le interpellanze di uno possono interessare anche l'altro (PANNIKAR, 2001). Questo è il tempo e lo spazio (post-modernità e piazza multicolore) dove le religioni possono concorrere a costruire un futuro per tutti, possono stare nello spazio pubblico non per rivendicare diritti del passato, ma per aiutare a costruire una grammatica di senso per la vita sul pianeta. Vogliamo, infondo, provare a trasformare problemi in occasioni di crescita scegliendo il pluralismo religioso non solo come realtà di fatto, ma come principio di un nuovo paradigma di convivenza ${ }^{1}$ (NASO; SALVARANI, 2015).

\section{Le interpellanze del pluralismo religioso al contesto occidentale....}

La crisi delle forme storiche delle religioni, in particolare quelle riferita alle chiese cristiane, protestanti, ma anche cattoliche, è una narrazione accolta da molta letteratura in occidente. Le indagini sociologiche, ma anche quelle delle scienze sociali che indagano le religioni, perfino i documenti magisteriali e pastorali descrivono la crisi dell'appartenenza religiosa, lo svuotamento delle

\footnotetext{
${ }^{1}$ Guida in questa riflessione è il recente volume di Naso; Salvarani (2015), da cui ricaviamo le principale considerazioni qui presentate.
} 
chiese, la messa in discussione della tradizione in campo etico... (ALDRIDGE 2005; BERGER, 1970; MATTEO, 2010). ${ }^{2}$ Eppure, la stessa letteratura avverte che il sacro non è morto, che, al contrario, si assiste ad un suo ritorno. Come stanno insieme, allora, crisi delle religione e vitalità del sacro? Un’interpretazione ormai matura è quella di coloro che sostengono di essere in epoca di post-secolarizzazione, dove "post" deve essere inteso come superamento di un paradigma (quello della secolarizzazione e della sua annunciata "morte di Dio") e introduzione in un altro modello interpretativo, dove "credere senza appartenere" sembra essere il modus dominante (CASANOVA, 2000; LENOIR, 2005; PACE, 1997). L'evidenza, poi, del pluralismo religioso nelle società occidentali diventa una realtà innegabile tanto è fatto quotidiano, pubblico e politico (ALLIEVI, 2010; JEKINS, 2009)³. Come tale pluralismo religioso in epoca post-secolare interpella il mondo occidentale? A noi sembra che tale realtà chieda all'occidente di vedere nella crisi delle forme storiche delle religioni un'opportunità spirituale: non è la ricerca di senso religioso che è in crisi, casomai i contenitori, le forme, i modelli storici che fin qui l'hanno accolta. Al mondo occidentale, il pluralismo religioso chiede di accettare come tempo favorevole quello della vulnerabilità delle istituzioni religiose, chiamate a rinnovarsi, a modificare la loro semantica, perfino ad accettare di mettere in discussione l'assolutezza dei propri messaggi religiosi (BECK, 2009; VATTIMO, 2002)4. Chiese e religioni come "ospedali da campo" (per rimandare ad una metafora di papa Francesco), capaci di accogliere la ricerca aperta e sofferta di tanti pellegrini, piuttosto che recinti dove confinare la salvezza divina, escludente chi si trova fuori da essi.

Un'ulteriore interpellanza del pluralismo religioso al contesto occidentale ci sembra quella di accettare fino in fondo l'evidenza della realtà: la fine della centralità dell'occidente nel mondo attuale. Visto soprattutto del versante delle religioni, tanto più quella cristiana, l'Europa non detiene più il monopolio religioso e occorre sapere "andare a sud". Significa lasciarsi interrogare dai laboratori

\footnotetext{
${ }^{2}$ Vedi oltre ad alcuni classici di riflessione sociologica quali, tra altri:Aldridge (2005); Berger (1970); anche la più recente analisi, soprattutto a livello giovanile, proposta dal pastoralista, Matteo (2010).

${ }^{3}$ Un'indagine sociologica a livello europeo sulla presenza pubblica delle religioni è quella offerta, per il caso del rapporto con il mondo islamico, da Allievi (2000), mentre rimane riferimento obbligato la ricerca offerta da Jekins (2009).

${ }^{4}$ Vedi una riflessione di tipo sociologico, Beck (2009). E una di carattere filosofico, Vattimo (2002).
} 
culturali, religiosi e spirituali che si compiono nel sud del mondo, dove la doppia appartenenza religiosa, il sincretismo culturale e religioso prima che essere una questione dottrinale, è stata e continua ad essere una questione vitale (JEKINS, 2004, 2008)5. Nuove forme religiose crescono e intendono partecipare all'eventuale Parlamento delle religioni, qualora fosse convocato. Ad esso, certo, viene invitato anche il mondo occidentale che però ha smesso di essere il centro della riflessione e vi partecipa come una regione del mondo, come una sua periferia.

\subsection{E al contesto italiano}

Come annunciato, il pluralismo religioso, quando declinato nello specifico, fa i conti con realtà storiche, come quella italiana, che si confrontano con proprie, singolari interpellanze. La prima di queste, infatti, è la scoperta sociologica recente per cui, con un efficace gioco di parole, non ci troviamo più davanti alla "religione degli italiani" quanto a "l'Italia delle religioni"(NASO; SALVARANI, 2009; PACE, 2004) ${ }^{6}$. Infatti, mentre per altri contesti europei (soprattutto anglosassoni o dei paese nordici), la diversità religiosa, almeno confessionale, è stata una realtà caratterizzante la storia di quei paesi, nel contesto italiano l'egemonia di una confessione religiosa, quella cattolica, ha dominato nel tempo. Scoprirsi ora a fare i conti con "l'Italia delle religioni", obbliga il cittadino italiano a riconoscere, se vuole accogliere l'appello della realtà, un nuovo pluralismo. Anche in Italia, infatti, la diversità rappresentata dalle storiche minoranze religiose, quella valdese e quella ebraica soprattutto, caratterizza la storia moderna del paese. Ma oggi la quantità e la qualità della differenza religiosa e culturale impegna a riconoscere un diverso pluralismo religioso che interroga la stessa tradizionale identità dell'italiano medio: egli, infatti, non trova più, volens nolens, una religione di riferimento, ma incontra un mercato religioso sempre più diversificato. Sapere, cioè, che un italiano può essere musulmano, senza smettere di essere italiano è una considerazione che, fino

\footnotetext{
${ }^{5}$ Qui il riferimento, anche se limitato alla realtà del cristianesimo, va ancora alla ricerca di Jekins (2004, 2008).

${ }^{6}$ Il titolo è suggerito dal volume a cura di Naso; Salvaran (2009). Vedi inoltre Pace (2004).
} 
a poco tempo fa, non era per niente pacifica (e ancora adesso per i populismi di tutti i segni politici).

Altra considerazione che emerge volendo interrogare lo specifico culturale italiano a partire dalla realtà del pluralismo religioso è quella che osserva, in apparente contraddizione con quanto prima affermato, la poca consistenza del mercato religioso in questo paese. Significa dire che a confronto con altre comunità e storie nazionali, la diversità religiosa in Italia rimane contenuta, almeno nei numeri se non nella tipologia (PACE, 2013). Le confessioni religiose, per rimanere in ambito cristiano, non hanno, cioè, visto crescere i loro membri; continuano ad essere piccole minoranze quelle degli italiani protestanti ed ortodossi. Invece, emerge sempre più, come indagato dai sociologi, una "religione all'italiana": dove, cioè, il pluralismo si da non fuori, ma dentro la propria confessione religiosa (GARELLI, 2011). Esiste sempre più diffuso un diverso e ricco modo di appartenere alla tradizione cattolica. Oggi, molto più di ieri, anche in Italia si può essere cattolici in modi diversi (CIPRIANO et al, 2003; CARTOCCI, 2011).

Altro discorso, quello di osservare le religioni arrivate in Italia con gli immigrati. Qui la complessità è fenomenica. E chiede di essere interpretata con adeguati strumenti (SALVARANI, 2014) 7. In ordine, ad esempio, alle diverse strategie messe in campo dalle differenti comunità religiose: per una comunità che rivendica e chiede integrazione, esiste anche quella che reclama la propria differenza ed identità. Sono dinamiche di tipo sociale, piuttosto che legate alla dottrina religiosa e sono trasversali le diverse comunità e generazioni di fedeli. L'interpellanza che nasce da tale presenza è quella, tra le altre, di aiutare a formare nuove leadership religiose capaci di postura laica e democratica. Non si risolvono i problemi di convivenza rimanendo al chiuso della propria tradizione (nel caso delle comunità reattive) oppure rinunciando alla propria identità (rischio di chi cerca l'integrazione senza negoziazione), ma rinnovando la propria formazione, anche religiosa, nel nuovo contesto storico e culturale in cui si vive. Ma per far questo,

\footnotetext{
${ }^{7}$ Per il contesto italiano rimane uno strumento di riferimento l'annuale dossier statistico curato da Caritas/Migrantes ed edito da IDOS, Roma che contempla dati anche in ordine all'appartenenza religiosa degli immigrati. Rimane vero che resta difficile avere un quadro statistico preciso rispetto alle religioni nel contesto italiano: vedi, al riguardo, Salvarani (2014).
} 
appunto, occorre investire nella formazione e qui il ritardo delle istituzioni e del mondo accademico rischia di essere una colpa: manca un investimento capace di produrre una nuova cultura interreligiosa per i "vecchi" e "nuovi” italiani.

Altro appello che possiamo raccogliere dal pluralismo religioso in Italia è quello di sapere promuovere la "pluralità qualitativa". Dove promuovere non significa semplicemente accogliere, ma soprattutto maturare e far fare un salto di qualità ai modi diversificati di vivere la stessa fede. Far diventare adulte quelle forme di "designer religion" osservate dagli analisti sociali (altri usano l'espressione di bricolage religioso) (BERZANO, 2014). Superare l'analfabetismo religioso che pesa soprattutto sulle nuove generazioni, senza più riferimenti ed anche aiutare a vivere la religione non solo come esperienza personale ed estetica, ma anche per il contributo collettivo e storico che essa può dare (MELLONI, 2014). Il mondo religioso carismatico, ad esempio, non è solo un fenomeno da osservare, ma anche da capire e da valorizzare quando aiuta a vivere la ricerca di senso. Così come cercano senso i tanti pellegrini sulle strade di Santiago di Compostela e dintorni. Tale "pluralità di ricerca" va valorizzata e suona come un ulteriore appello proveniente dal pluralismo religioso esistente dentro la stessa tradizione confessionale.

Il pluralismo religioso italiano invita, inoltre, a decostruire l'idea di unità. Prima ancora che un problema di ordine filosofico (per cui, diversamente dalla filosofia classica, occorre distinguere e non rendere sinonimi Unum et Bonum), l'appello derivante dalla diversità religiosa presente nel contesto italiano è quello di saper rispettare e valorizzare le differenze, quello di non accettare di avere un'unica parola sui temi e problemi. Il guadagno di tale "decostruzione" investe direttamente il concetto di laicità (NASO, 2007). Ad una comunità che "agisce" in modo plurale e non si limita ad affermare la bontà della differenza culturale e religiosa, sarà più facile ripensare l'idea di laicità oltre l'interpretazione che la riduce a separazione tra comunità statale e comunità religiosa. Se la pluralità religiosa diventa uno stile e non si ferma a dichiarazione, allora anche la laicità 
potrà arricchirsi del contributo delle diverse grammatiche religiose sulla vita e il suo senso, allora anche le religioni potranno partecipare al dibattito sulle politiche pubbliche e in generale sulla vita del paese.

A questo livello del discorso, il pluralismo religioso che si dà in Italia porta in dote un'interpellanza importante. La Costituzione italiana, nel suo articolo ottavo, prevende, infatti, l'istituto delle intese, come forma giuridica capace di riconoscere e valorizzare le comunità religiose diverse dalla cattolica (con la quale, invece, vige l'istituto del concordato). Pur riconoscendo, in un'impostazione di questo tipo, una distinzione gerarchica poco "democratica”, occorre invece valorizzare il dispositivo giuridico e politico delle intese tra Stato e religioni (FERRARI, 2013). Il pluralismo culturale e religioso, infatti, ha storicamente ricevuto da parte degli stati europei due risposte: quella espressa dall'universalismo alla francese o quella messa in campo dal comunitarismo di tipo anglosassone. Oggi occorre riconoscere che entrambe queste modalità non soddisfano il rapporto con la diversità: se la prima fatica a riconoscere la specificità religiosa (al punto che il velo è vietato nelle scuole pubbliche francesi), la seconda ha messo in moto dinamiche sociali e spirituali resistenti all'integrazione (come testimonia purtroppo il fenomeno dei foreign fighter molti dei quali nati nel Regno Unito). La via italiana al pluralismo religioso rappresentata dalle intese potrebbe, invece, proporsi come una diversa possibilità di governance della multireligiosità capace di tenere insieme laicità, rispetto dei diritti e dei doveri, riconoscimento pubblico della comunità di fede, trasparenza organizzativa della stessa e stimolo a relazioni istituzionali costruttive (e non solo rivendicative). Certo, per far questo, c’è bisogno di superare quella lettura circa il mondo dell'immigrazione che interpreta l'immigrato come "figura espiatoria": colpevole dei mali della società che ha voluto o piuttosto dovuto accoglierlo. Se una Regione (ci riferiamo alla Lombardia) ha potuto emanare, nel gennaio del 2015, un provvedimento bcosiddetto “anti-moschee” è proprio in forza di questa lettura colpevolizzante l’immigrato e la sua diversità anche religiosa. Tipico caso, direbbero gli antropologi, dove il diverso diventa l'opposto. E con questo prende il via la demonizzazione dell'altro in quanto altro. E' l'approccio etnico-tribale alla questione del rapporto con l'altro che vale anche in caso di diversità religiosa. 
Infine, un ultimo appello che possiamo raccogliere dal pluralismo religioso presente in Italia è quello della valorizzazione del carattere popolare del dialogo interreligioso. I colpevoli ritardi delle istituzioni, l'ambiguità della politica, il poco o nullo investimento culturale sul tema da parte delle università, le prudenze timorose delle comunità ecclesiali (anche nel ripensare, a partire dal pluralismo, la propria pratica pastorale), non possono, però, nascondere la ricchezza di iniziative, attività e proposte che si danno sul territorio italiano (DAL CORSO, 2014). Protagoniste associazioni, gruppi, realtà di volontariato, centri di formazione, il dialogo interculturale ed interreligioso gode oggi in Italia di un'agenda ricca e aperta tutto l'anno. Esiste, cioè, un carattere popolare del dialogo interreligioso che chiede di essere valorizzato, anche perché è quello a cui arriva la gente . La frequentazione ai vari festival, incontri e convegni che mettono a tema, spesso in modo esperienziale oltre che culturale, il tema della diversità concorre a fare del dialogo interreligioso non solo un tema, ma un'esperienza. Perché è proprio vero: non esiste il dialogo interreligioso, quello che esiste è il dialogo tra persone di diverse appartenenze religiose e spirituali. Questo vale per le piazze, ma certo per i social network ed ancora di più per la scuola (TRIDENTE, 2014; SALVARANI, 2006).

Resta che per riconoscere la "verità teologica" del pluralismo, dopo averne raccolto le interpellanze, serve una diversa teologia del dialogo. Per decostruire l'idea di unità, ma anche per valorizzare pastoralmente la pluralità interna e qualitativa, per scegliere risolutamente nuove forme giuridiche capaci di rispettare l'altra comunità di fede, per valorizzare i percorsi popolari e di base presenti nell'Italia delle religioni, abbiamo bisogno di una diversa riflessione sul dialogo interreligioso. Una teologia post-moderna del dialogo.

\section{Per una teologia post-moderna del dialogo}

Se la filosofia post-moderna della differenza ha sollevato la questione della de-costruzione come condizione metodologica per il pensiero critico, anche la 
teologia in tempi post-moderni, fedele alla sua dimensione profetica, è chiamata a prendere sul serio l’irriducibilità della differenza. E da essa imparare il dialogo.

Per la teologia, allora, la post-modernità, tra contributi e possibili derive, significa operare una critica ai sistemi totalizzanti, anche quello a carattere religioso. Il dialogo, qui, non può essere quello della diplomazia, strategia politicamente corretta, ma deve essere quello dell'ontologia: siamo tutti in ricerca perché "mancanti”, nessuno è proprietario della verità. I sistemi totalizzanti, infatti, finiscono per essere totalitari, contrari al dialogo. Ma assumere il paradigma della post-modernità per la teologia significa anche un superamento della visione di religione come sistema arcaico e sacrificale. Le religioni abitano il tempo che viviamo come grammatiche sulla vita e non come retaggio del passato, col destino segnato. Per questo possono non solo dialogare tra loro, ma anche con la politica: voce, quella delle religioni, che può contribuire alla costruzione della polis. La dimensione post-secolare della seconda modernità lascia spazio alle religioni.

Una teologia post-moderna, poi, davanti alla violenza imperante annuncia il carattere alternativo del perdono, capace di aprire il dialogo anche là dove, vincendo il rancore, esso sembrava impossibile. E finalmente, la teologia della postmodernità è quella in cui la vittima, passando per un'esperienza estrema, è capace, anche così, di testimoniare una speranza possibile. Tale teologia, infatti, ha trasformato il dogma antico: ora "extra victimas nulla salus"(ALVAREZMENDONZA, 2011) ${ }^{8}$. Il dialogo interreligioso diventa un dialogo soteriologico e non più dogmatico.

Riprendendo le potenzialità di tale teologia post-moderna del dialogo, occorre subito ricordare, a proposito di pensiero critico, che la tensione, la preoccupazione per l'esistenza che le religioni portano in dote aiuta nel decostruire i sistemi linguistici e tanto più politici e ideologici chiusi. Vivere per le religioni è “ex-istere": vivere per gli altri. L’assoluto non è mai nel sistema, sia esso quello della tecnica, della politica o anche della morale religiosa. Per le religioni, con le

${ }^{8} \mathrm{E}^{\prime}$ la feconda definizione che offre il teologo messicano Alvarez- Mendonza (2011). A cui le riflessioni di questa parte sono debitrici. 
parole di un vescovo brasiliano, "solo Dio e la fame sono assoluti, tutto il resto è relativo" (Pedro Casaldaliga). Il dialogo interreligioso si può costruire condividendo tale visione critica nei confronti di tutti i sistemi che si presentano con il carattere dell'assolutezza. Il carattere decostruttivo e critico è metodo anche della teologia post-moderna.

Attraverso tale metodo, essa può mettere in discussione il racconto narcisistico moderno-occidentale: quello del mercato dove fare il proprio interesse smette di essere vizio, peccato e diventa virtù. Il disinteresse piuttosto che l'interesse è la categoria alternativa che la teologia post-moderna propone. La narrazione biblica, ma anche quella coranica, la compassione buddista, ma anche la saggezza confuciana sanno che il dono e la donazione sono il cuore mistico della realtà. Ben oltre la relazione interessata, del "do ut des”, foriera di violenza, proposta dal mercato moderno.

Ora, la teologia post-moderna apre al dialogo anche perché smaschera il carattere onnipotente del soggetto moderno. Esso, nel suo desiderio di onnipotenza, finisce per manipolare le relazioni: il mondo diventa oggetto del soggetto padrone, gli altri non possono essere riconosciuti nella loro diversità da me dominatore e infine Dio posso pensarlo solo come giudice e vendicatore come sono io che comando. Le relazioni risultano imprigionate, il dialogo impossibilitato. Invece, la teologia che apre al dialogo è quella che "ragiona" a partire dalle vittime, annunciando il perdono piuttosto che il risentimento e vedendo nell'altro il fratello piuttosto che il lupo.

A rompere il circolo mimetico e violento della società attuale, può, allora, contribuire quella riflessione teologica all'altezza dei tempi che pensa e agisce a partire dalla "forza della debolezza". Il perdono invece che la vendetta, aiuta a vivere la relazione non nello spirito di rivalità risentita, ma in quello della relazione dentro la differenza. Qui il dialogo diventa possibile perché libero dalla violenza. E anche la verità delle religioni appare “discreta”. La discrezione è il tratto dialogante. 
La religione e la riflessione su di essa in tempi post-moderni oltre al carattere critico e decostruttivo, porta in dote anche una grammatica della diversità. Liberata dalla tentazione sacrificale della modernità, dove la competizione, la rivalità, il risentimento sembrano essere i tratti dominanti, la teologia post-moderna, in fedeltà al suo patrimonio di riflessione e di pratica, pronuncia e vive una "grammatica escatologica" che apre al dialogo. A partire, cioè, da una nuova intelligenza sulla realtà che lo sguardo della vittima, libera dal rancore e disposta al perdono, offre. Il Regno di cui parla tale teologia, allora, è una realtà dinamica, disposta ad accogliere anche altre utopie come sono il paradiso musulmano, il nirvana buddista. E il futuro sognato dalla vittima è un desiderio liberato dal mimetismo, spazio simbolico e insieme reale da vivere non contro l'altro, ma insieme all'altro e infine il perdono a cui la vittima perdonante richiama apre un presente nuovo, dove l'ideologia del nemico, dell'infedele, dell'eretico non può essere coltivata, alimentata.

Davanti al mondo così com'è si apre la possibilità di vivere relazioni gratuite: e il mondo fratricida può essere luogo di compassione. Non solo la misericordia, ma la gratuità libera le relazioni. La riflessione sulla fede in tempi post-moderni, allora, è "fides quaerens gratuitatem" (fede che cerca la gratuità). La diversità, cioè, è accolta non solo come esigenza etica, qui l'amore, ma anche come imperativo teologico: vedere la realtà a partire dall'altra storia, quella delle vittime. Quella della teologia del dialogo in epoca post-moderna, allora, è una diversità trascendente, viene da un'altra parte, viene da oltre. Non si tratta solo di una politica attenta alle minoranze, si tratta di uno sguardo diverso sulla realtà. Qui il carattere teologico della diversità.

Siamo di fronte, cioè, ad un'intelligenza teologica del reale che sa vedere l'immanenza come via alla trascendenza come ci ricordano i padri: "gloria Dei vivens homo" (Ireneo di Lione). Non la via della ragione, non quella della natura, ma la via di una vita liberata dalla violenza, dall'interesse, in fondo dall'ego. Qui le religioni concordano e il dialogo che ne esce è quello di una diversa interpretazione del reale. Questo non significa che la teologia post-moderna del dialogo non sappia 
riconoscere il carattere conflittuale della società in cui vive. Tra la rivalità narcisistica che sembra presiedere i rapporti e piccole esperienze etiche dove riconoscere l'altro non più come rivale, ma come compagno di viaggio, la teologia del dialogo mentre non accetta che la realtà così com'è sia il senso ultimo del reale, si impegna a costruire, già qui, una comunità non dominata dal risentimento, dove la differenza abbia spazio. Le identità mimetiche, reattive, improprie possono diventare identità plurali ed ospitali. La categoria fondamentale di tale teologia del dialogo, allora, è quella della gratuità.

\section{Conclusioni}

Assumere la gratuità e dell'ospitalità come categoria fondante significa ripensare e praticare in altro modo il dialogo inter e intra-religioso. Al centro dello stesso, cioè, non stanno i sistemi dogmatici, preoccupati di dare credibilità alla fede, come non si trovano gli impianti ecclesiologici, le realtà istituzionali anch'esse tese a giustificare la própria credibilità davanti al mondo. Questi sonotemi secondi. Il dialogo, invece, diventa autentico quando mette al centro la grammatica sulla gratuità come senso ultimo della realtà. L'illuminazione buddista, la misericordia musulmana, la carità cristiana, l'ospitalità ebraica sono esperienze gratuite. Sanno dire la trascendenza dentro l'immanenza (per questo sono esperienze religiose) non a partire da una grammatica razionale, ma dal principio gratuità. La logica della vita donata, irrazionale e immorale per la visione dominante, presiede l'intelligenza sul reale delle diverse religioni. Qui il dialogo acquista fondamento. Non solo una vita donata, ma anche una vita perdonata: un nuovo dono, quella di una relazione che non si chiude nel risentimento. E il perdono è parola religiosa per eccellenza; appartiene, anche se spesso tradita, al linguaggio delle religioni. E' il loro modo di vedere i rapporti. Infine, lo sguardo della gratuità, tra dono e perdono, offre anche un nuovo modo di stare nel fallimento, nella crisi: l'avvenire è una promessa divina, dicono le religioni abramitiche, ma anche tutto è impermanente e quindi passa, il presente non ci schiaccia, ripetono le spiritualità orientali. Insomma, la teologia post-moderna è dialogica perché narra la trascendenza a partire dall'esperienza 
della gratuità, dell'ospitalità. E oltre al divino, anche l'umano può fare una diversa esperienza di incontro con l'altro: scoprendosi vulnerabile, sa di non essere al centro della realtà, ma bisognoso e capace di rispondere ai bisogni dell'altro. Se ospitato, allora, la persona umana può imparare che l'altro-nemico diventa, invece, fratello e che più della reciprocità (do ut des) vale la gratuità, il disinteresse, l'asimmetria per vivere nuove relazioni. E se il trascendente è mistero di donazione questo non vale tanto per l'aldilà, ma giudica l'aldiqua: vivere gratuitamente, vivere secondo la logica dell'ospitalità è segno di una vita secondo il volere divino. Diventa possibile abitare diversamente il mondo, fare delle finitudine non un limite, ma il senso ultimo del vivere, perché consapevoli che prima di pensare, siamo stati pensati.

\section{RIFERIMENTI}

ALDRIDGE, Alan. La religione nel mondo contemporaneo. Una prospettiva sociologica. Bologna: Il Mulino, 2005.

ALLIEVI, S. La guerra delle moschee. Venezia: Marsilio, 2010.

ALVAREZ- MENDOZA, C. Deus absconditus. Dèsir, memorie et imagination eschatologique. Essai de theologie fondamentale postmoderne. Parigi: Editon du Cerfi, 2011.

ALVAREZ-MENDOZA,Carlos. Deus absconditus. Dèsir, memorie et imagination eschatologique. Essai de theologie fondamentale postmoderne. Parigi: Editon du Cerf, 2011.

BECK, U. Il Dio personale. La nascita della religiosità secolare. Roma-Bari: Laterza, 2009

BERGER, P.L. Il brusio degli angeli. Il sacro nella società contemporanea. Bologna: Il Mulino, 1970.

BERZANO, L.; NUEVO, J.A. Designer Religion . Disponibile in: $<$ www.designerreligion.wordpress.com 2014>.

BERZANO, L. Spiritualità senza Dio?. Milano: Mimesis, 2014

CARTOCCI, R. Geografia dell'Italia cattolica. Bologna: Il Mulino, 2011.

CASANOVA, J. Oltre la secolarizzazione. Le religioni alla riconquista della sfera pubblica. Bologna: Il Mulino, 2000. 
CIPRIANO, R; GUIZZARDI, G; PACE, E. Un singolare pluralismo. Indagine sul pluralismo morale e religioso degli italiani. Bologna: Il Mulino, 2003.

DAL CORSO, M. Strumenti di cura dell'analfabetismo religioso: le esperienze dal basso. In: MELLONI, A. (a cura di). Rapporto sull'analfabetismo religioso in Italia. Bologna: Il Mulino, 2014.

FERRARI, A. La libertà religiosa in Italia: un percorso incompiuto. Roma: Carocci, 2013

GARELLI, F. Religioni all'italiana. L’anima del paese messa a nudo. Bologna: Il Mulino, 2011.

JEKINS, P. Il Dio dell'Europa. Il cristianesimo e l'islam in un continente che cambia. Bologna: EMI, 2009.

JEKINS, P. I nuovi volti del cristianesimo. Milano: Vita e Pensiero, 2008.

JEKINS, P. La terza Chiesa. Il cristianesimo nel XXI secolo. Roma: Fazi editore, 2004

LENOIR, F. Le metamorfosi di Dio. La nuova spiritualità occidentale. Milano:

Garzanti, 2005.

MATTEO, A. La prima generazione incredula. Soveria Manelli: Rubettino, 2010.

MELLONI, A. (a cura di), Rapporto sull'analfabetismo religioso in Italia. Bologna: Il Mulino, 2014.

NASO, P. Laicità. Bologna: EMI, 2007

NASO, P; SALVARANI, B. (a cura di). I ponti di Babele: cantieri, progetti e criticità nell'Italia delle religioni.Bologna: EDB, 2015.

NASO; SALVARANI. Il muro di vetro. L'Italia delle religioni, I Rapport. Bologna: EMI, 2009.

PACE, E. (a cura di). Le religioni nell'Italia che cambia. Mappe e bussole. Roma: Carocci, 2013.

PACE, E. (a cura di). Le religioni nell'Italia che cambia. Mappe e bussole. Roma: Carocci, 2013.

PACE, E. Credere nel relativo. Persistenze e mutamenti nelle religioni contemporanee. Torino: UTET, 1997.

PACE, E. Vecchi e nuovi dei: la geografia religiosa dell'Italia che cambia. Milano: Paoline, 2004. 
PANIKKAR, R. L'incontro indispensabile: il dialogo delle religioni. Milano: Jaca Book, 2001.

SALVARANI, B. Educare al pluralismo religioso. Bologna: EMI, 2006.

SALVARANI, B. I difficili numeri delle religioni in Italia. In: MELLONI, A. Rapporto sull'analfabetismo religioso in Italia. Bologna: Il Mulino, 2014.

SALVARANI, B. Vocabolario minimo del dialogo interreligioso. Per un'educazione al dialogo tra le fedi. Bologna: EDB, 2008.

TRIDENTE, G. (a cura di). Teoria e pratica del giornalismo religioso. Roma: EDUSC, 2014.

VATTIMO, G. Dopo la cristianità. Per un cristianesimo non religioso. Milano: Garzanti, 2002. 